\title{
Bioluminescence and second harmonic generation imaging reveal dynamic changes in the inflammatory and collagen landscape in early osteoarthritis
}

\author{
Carrie K. Hui Mingalone ${ }^{1} \cdot$ Zhiyi Liu $\mathbb{C}^{2,3} \cdot$ Judith M. Hollander ${ }^{1} \cdot$ Kirsten D. Garvey $^{4}$ - Averi L. Gibson ${ }^{1}$. \\ Rose E. Banks ${ }^{4} \cdot$ Ming Zhang ${ }^{5} \cdot$ Timothy E. McAlindon ${ }^{5} \cdot$ Heber C. Nielsen $\mathbb{D}^{1} \cdot$ Irene Georgakoudi $^{2} \cdot$ Li Zeng $^{1,4,6}$
}

Received: 8 September 2017 / Revised: 12 December 2017 / Accepted: 14 December 2017 / Published online: 14 March 2018

(c) United States \& Canadian Academy of Pathology 2018

\begin{abstract}
Osteoarthritis (OA) is a leading cause of chronic disability whose mechanism of pathogenesis is largely elusive. Local inflammation is thought to play a key role in OA progression, especially in injury-associated OA. While multiple inflammatory cytokines are detected, the timing and extent of overall inflammatory activities in early OA and the manner by which joint inflammation correlates with cartilage structural damage are still unclear. We induced OA via destabilization of the medial meniscus (DMM) in NFKB luciferase reporter mice, whose bioluminescent signal reflects the activity of NFKB, a central mediator of inflammation. Bioluminescence imaging data showed that DMM and sham control joints had a similar surge of inflammation at 1-week post-surgery, but the DMM joint exhibited a delay in resolution of inflammation in subsequent weeks. A similar trend was observed with synovitis, which we found to be mainly driven by synovial cell density and inflammatory infiltration rather than synovial lining thickness. Interestingly, an association between synovitis and collagen structural damage was observed in early OA. Using Second Harmonic Generation (SHG) imaging, we analyzed collagen fiber organization in articular cartilage. Zonal differences in collagen fiber thickness and organization were observed as soon as OA initiated after DMM surgery, and persisted over time. Even at 1-week post-surgery, the DMM joint showed a decrease in collagen fiber thickness in the deep zone and an increase in collagen fiber disorganization in the superficial zone. Since we were able detect and quantify collagen structural changes very early in OA development by SHG imaging, we concluded that SHG imaging is a highly sensitive tool to evaluate pathological changes in OA. In summary, this study uncovered a dynamic profile of inflammation and joint cartilage damage during OA initiation and development, providing novel insights into OA pathology.
\end{abstract}

These authors contributed equally: Carrie K. Hui Mingalone, Zhiyi Liu.

Electronic supplementary material The online version of this article (https://doi.org/10.1038/s41374-018-0040-9) contains supplementary material, which is available to authorized users.

Li Zeng

Li.Zeng@tufts.edu

1 Program in Cell, Molecular, and Developmental Biology, Sackler School of Graduate Biomedical Sciences, Tufts University, Boston, MA 02111, USA

2 Department of Biomedical Engineering, Tufts University, Medford, MA 02155, USA

3 Wellman Center for Photomedicine, Massachusetts General

\section{Introduction}

Osteoarthritis $(\mathrm{OA})$ is a widespread debilitating disease that has no cure [1-3]. OA is characterized by joint cartilage destruction and loss of cartilage matrix, as well as pathological changes of other joint tissues, such as synovial inflammation and subchondral bone sclerosis $[4,5]$. Current treatments generally rely on symptom relief, rather than

Hospital, Harvard Medical School, Boston, MA 02114, USA

4 Department of Immunology, Tufts University School of Medicine, Boston, MA 02111, USA

5 Division of Rheumatology, Tufts Medical Center, Boston, MA 02111, USA

6 Department of Orthopaedics, Tufts Medical Center, Boston, MA 02111, USA 
disease modification. To design strategies to slow down $\mathrm{OA}$, it is important to understand the mechanisms that underlie the initiation and progression of this disease. However, pathological changes in early stages of OA tend to be subtle and often elude investigation. In recent years, some of these changes have been discovered with the use of advanced imaging technologies [6]. For example, fibrillation of the cartilage surface that was not visible by eye could be observed using optical coherence tomography, and alterations in mechanical properties were discovered using atomic force microscopy (AFM) [7-10]. Additionally, loss of glycosaminoglycan (GAG) content and water retention could be detected by AFM or magnetic resonance imaging and Fourier transformed infrared imaging [11-13]. Quantitative polarized light microscopy has been employed to visualize collagen, taking advantage of collagen's birefringence [7, 14-17]. However, collagen structural changes in early stages of OA have not been systematically investigated, even though collagen is a key component of the cartilage matrix.

Parallel to structural alterations, biochemical changes take place in early OA [18-20]. One such aspect of biochemical change is inflammation, which is especially pronounced in injury-induced OA $[9,21]$. Inflammation has been associated with pathological changes in the extracellular matrix in many diseases causing extracellular matrix (ECM) remodeling, including fibrosis and matrix degradation [22-25]. In cartilage, pro-inflammatory cytokines, such as IL- $1 \beta$ and TNF $\alpha$, can initiate a cascade of catabolic events [26]. A key step in this cascade involves the activation of the nuclear factor kappa $\mathrm{B}(\mathrm{NF \kappa B})$ pathway. NFkB subsequently induces the expression of catabolic genes, such as matrix metalloproteinases (MMPs). These MMPs cleave cartilage ECM components, causing cartilage destruction and OA [27, 28]. On the other hand, cytokines known to have anti-inflammatory activities, such as IL-10 and IL-1Ra, are also induced following joint injury [29-32]. The overall inflammatory activity from these stimuli during the course of early OA development and the effects on joint pathology are still not clearly understood.

In this study, we employed two imaging tools to investigate early inflammatory and collagen changes in the joint of a widely used mouse experimental OA model, the destabilization of the medial meniscus (DMM). We tracked the activity of NFKB, a key mediator of inflammation [33], through live bioluminescence imaging of luciferase activity in NFkB reporter mice during early OA development. In addition to evaluating synovial inflammation and cartilage GAG loss, we also studied collagen fiber structural changes by Second Harmonic Generation (SHG) imaging, a powerful two-photon imaging technique ideal for imaging of non-centrosymmetric molecules such as collagen fibers [34-36]. SHG imaging allows for high-resolution assessment of several collagen fiber properties, including thickness and orientation [34, 37, 38]. It involves the simultaneous interaction of two photons with a molecule, leading to the scattering of a single photon that possesses twice the energy, and therefore, half the wavelength, of the incident photons. This is a coherent process, which requires that the scattering molecule lacks a center of symmetry for net second harmonic signal generation. This is the reason for which collagen fibers are specific sources of particularly strong SHG signal in cartilage ECM. By using bioluminescence and SHG imaging in this study, we observed a dynamic change of NFKB activity and synovial inflammation, as well as distinct zonal alteration of collagen organization in articular cartilage in the early stages of $\mathrm{OA}$ development.

\section{Materials and methods}

\section{Experimental animals}

All animal care and experimental procedures were approved by the Institutional Animal Care and Use Committee at Tufts University (B2016-177). Mice were caged under standard light-dark cage conditions. The NFKB (Balb/C) reporter mouse NFKB-RE-luc, consisting of $6 \mathrm{NFKB}$ response elements, was purchased from Taconic (NY, USA). Destabilization of the medial meniscus (DMM) surgery was performed on 8-week-old male mice according to established protocol [39-41]. Briefly, the right knee joint was opened along the medial border of the patellar ligament and the medial meniscotibial ligament was severed. The left knee joint received a sham surgery, in which the ligament was exposed, but not severed.

\section{In vivo NFKB bioluminescence imaging}

To visualize NFkB bioluminescence, the mouse knee (i.e., stifle joint) was shaved and a skin incision was created. Mice were serially imaged using the Xenogen IVIS 200 Biophotonic Imager (PerkinElmer) at the same hour of the day at $1,3,7,11,13,15,17$, and 19-weeks post-surgery with 3-4 mice per time point. Before each imaging session, a baseline bioluminescent signal was measured. Then, $50 \mathrm{ng}$ of Luciferin (Goldbio) was intra-articularly injected through the patellar ligament with a 30-gauge needle, and another bioluminescent image was acquired 5 min later. As controls, non-surgery age-matched mice were also imaged. Signal intensity was quantified using Living Image (PerkinElmer). A region of interest was drawn over each knee and the mean signal radiance was measured. The pre-injection signal was subtracted as background from the signal detected following luciferin injection. 


\section{Histological analysis}

Isolated knee joints were fixed in $4 \%$ paraformaldehyde overnight and then decalcified in 10\% EDTA. Samples were embedded in paraffin and sagittally sectioned at $5 \mu \mathrm{m}$ thickness. Sections were stained with $0.1 \%$ Safranin O (Sigma-Aldrich) to assess glycosaminoglycan (GAG) content and counterstained with Gill's hematoxylin (Ricca Chemical Company) and $0.02 \%$ Fast Green (Sigma-Aldrich). Articular cartilage damage was blindly scored using a modified Mankin system to account for Safranin O staining loss and articular cartilage structural damage [42-44]. With respect to Safranin $O$ staining loss, a score of 0 indicates no staining loss. Scores of 1 to 6 indicate partial staining loss in varying areas of cartilage, while scores 7 to 12 indicate complete loss of staining in varying percentage areas of cartilage. With respect to cartilage structural damage, a score of 1 to 6 indicates minor damage of the structure in less than half of the articular surface in varying percentage areas of cartilage (less than or greater than $1 / 2$ of the cartilage surface, less than or greater than $1 / 2$ of the cartilage depth), while higher scores (i.e., 7 to 12) indicate severe and widespread damage of the structure $[42,44]$. Then scores of cartilage matrix loss and structural damage were added. While tidemark duplication, fibrocartilage, chondrocyte clones, hypertrophic chondrocyte, and subchondral bone changes are all part of OA pathology, these features were less obvious in our time frame of study $[42,44]$. Thus, OA score was mainly driven by Safranin O and articular cartilage damage scores. Synovitis severity in the anterior and posterior parts of the joint was determined following established methods, in which factors contributing to synovitis (resident cell density, inflammatory cell infiltrates, and synovial lining thickness) were each assigned a specific point from 0 to 3 , with 3 being the most severe [ 45 , 46]. The sum of all three categories was calculated as the total synovitis score.

Immunohistochemistry (IHC) for collagen I, collagen II, collagen $\mathrm{X}$, and macrophages (F4/80) was performed on serial sections from the same joints used for Safranin O staining [40, 46, 47]. For antibodies used and antigen retrieval methods, refer to Supplemental Table 1. The percentage of $\mathrm{F} 4 / 80(+)$ cells in the inner synovial lining (3-4 layers) were scored blindly and quantified (ImageJ, NIH). Sections without primary antibody incubation served as negative controls for staining.

\section{Light microscopy}

Bright field or fluorescent images were taken on an Olympus IX-71 microscope, using the Olympus DP70 or DP80 digital cameras. The optical parameters and camera exposure time were kept constant among samples of the same experiment.

\section{Second harmonic generation (SHG) imaging and two-photon excited fluorescence (TPEF) imaging}

Joints were embedded in OCT without fixation or decalcification, and cryo-sectioned at $40 \mu \mathrm{m}$ thickness. Sections were collected with cryofilm type-IIc-[10] (Section-Lab Co, Japan) to preserve tissue integrity. Sections were rehydrated for $15 \mathrm{~min}$ in PBS before imaging. SHG and TPEF imaging were performed at the same time using a Leica TCS SP2 confocal microscope equipped with a tunable $(710-920 \mathrm{~nm})$ titanium-sapphire laser (Mai Tai; Spectra Physics). For both imaging procedures, an excitation of $800 \mathrm{~nm}$ was used. For SHG imaging, photons emitted at $400 \pm 10 \mathrm{~nm}$ were detected using a non-descanned photomultiplier tube detector. Endogenous TPEF emitted by chondrocytes was detected at $525 \pm 25 \mathrm{~nm}$ [48-52]. A water-immersion $63 \mathrm{x}$ objective (NA 1.2; $220 \mu \mathrm{m}$ working distance) was used for articular cartilage imaging. Both forward and backward SHG signals were collected for analysis in MATLAB.

Each SHG cartilage image was separated into the superficial, transitional, and radial zones. These zones were delineated based on the following well-established notion: in the superficial zone, fibers and the long axis of chondrocytes lie parallel to the articular surface; in the radial zone, fibers and the long axis of chondrocytes lie perpendicular to the articular surface; in the transitional zone, fibers are sinusoidal [53-56]. The demarcation of the radial zone and the calcified cartilage zone was determined by the presence of the endplate, which presented itself as a dark line under SHG imaging. To assess relative thickness of the collagen fibers, the forward/backward ratio of SHG intensity was calculated following established methods [17, 3438]. To quantify the orientation and organization of the collagen fibers, we used algorithms we established previously [57] to analyze the FSHG images. A weighted vector summation approach was used first to assign a dominant orientation of the fiber(s) crossing each pixel. This orientation information could thus be displayed on a per pixel basis as a distribution for a given field of view to characterize the overall organization of the fibers and identify the presence of any dominant orientation angles. These distributions were then used to calculate the directional variance of the fibers within a field, as a quantitative metric of fiber organization. The value of the directional variance varies between 0 and 1 , with 0 indicating perfectly parallel fibers, and 1 corresponding to completely random organization $[48,58]$.

\section{Statistical analysis}

Data are shown as mean \pm SEM. Non-parametric data from Mankin scoring and synovitis scoring were evaluated via Kruskal-Wallis analysis followed by Dunn's multiple 


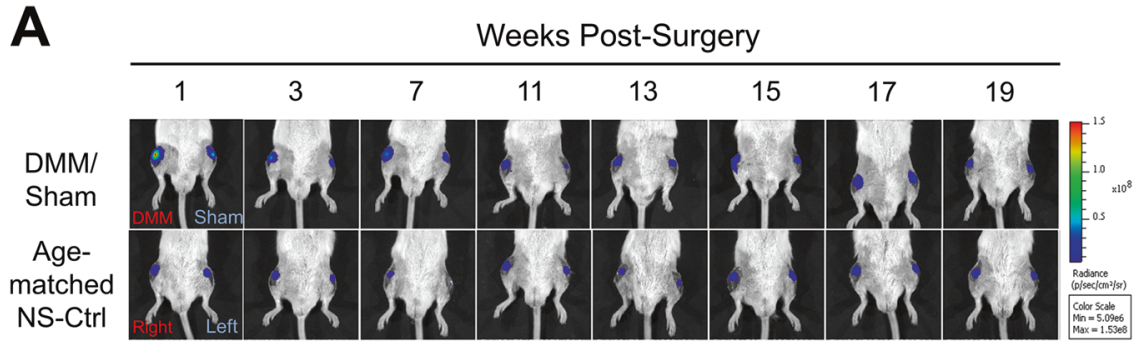

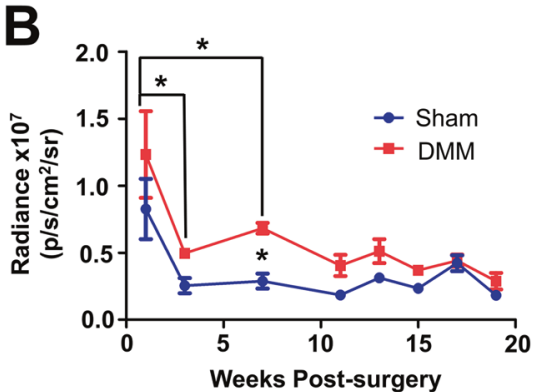

Fig. 1 Bioluminescence imaging demonstrating dynamic changes of

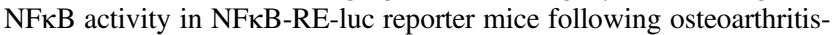
inducing surgery. a Images of in vivo bioluminescence imaging at multiple time points following DMM and sham surgery on the right and left knees of the mice, respectively. As controls, left and right knees of age-matched non-surgery mice were also imaged. At least 3 mice were used for each group. b Quantification of NFkB signal detected from serial bioluminescence imaging. c Quantification of

comparison test. All other experiments were evaluated with ANOVA followed by Bonferroni's multiple comparison test. Spearman's correlation was performed for correlation analysis. All statistical analysis was performed using Prism (version 5.01; GraphPad). Significant outliers ( $>3$ standard deviation away from the mean) were detected using the QuickCalcs outlier calculator (GraphPad) for parametric data, and custom $\mathrm{R}$ code for box plot detection of outliers ( $>1.5$ interquartile range) for non-parametric data ( $\mathrm{R}$ 3.3.2, $\mathrm{R}$ foundation) and removed from analysis. A $p$-value of 0.05 or less was considered significant.

\section{Results}

\section{A dynamic inflammatory profile in early $O A$ development}

To directly track inflammatory activity in the early stages of $\mathrm{OA}$, we performed DMM surgery on NFKB-RE-luc reporter mice, and monitored luciferase intensity in the knee joint as a readout for inflammation in live mice from 1 week to 19 weeks post-surgery [59-61]. For each mouse, the right knee was subjected to DMM surgery; the left knee was subjected to sham surgery, which served as an internal control for NFKB activity. High levels of bioluminescent

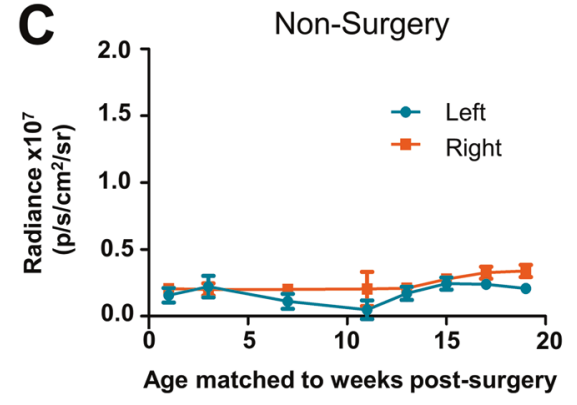

$\mathrm{NF \kappa B}$ signal detected from serial bioluminescence imaging in agematched non-surgery controls. For statistics, ANOVA analysis was conducted, followed by Bonferroni post-hoc test. The value at 1 week post-surgery was significantly higher than those of 3 weeks and 7 weeks post-DMM. Additionally, the value at 3 weeks in the DMM sample was significantly higher than that of the sham. Asterik $(*)$ denotes $p \leq 0.05$

signals were observed at 1 week following surgery, but there was no difference in signal intensity between the sham and the DMM joints (Fig. 1a, b), suggesting that the initial surge of inflammation likely resulted from the knee incision itself during both DMM and Sham surgery. By 3 weeks post-surgery, signals from the sham joint returned to levels similar to those of the non-surgery age-matched controls; in contrast, signals from the DMM joint, although reduced, remained higher than the sham signals until 7 weeks postsurgery. This indicates that destabilization of the knee joint through ligament transection induces a prolonged inflammatory phase, likely from the trauma of ambulation with the destabilized joint. After 7 weeks, the bioluminescent signals of both the DMM and the sham joints were further reduced to levels similar to those in non-operated controls (Fig. 1a, c), suggesting eventual resolution of acute inflammation after joint injury.

\section{Increased synovitis and subsequent induction of cartilage damage in early $O A$}

Since synovitis is known to be associated with inflammation and OA, we determined the level of synovitis in the weeks after surgery using an established synovitis scoring system [45, 61, 62]. This system accounts for the resident synovial cell population, the infiltrating 
A

Anterior

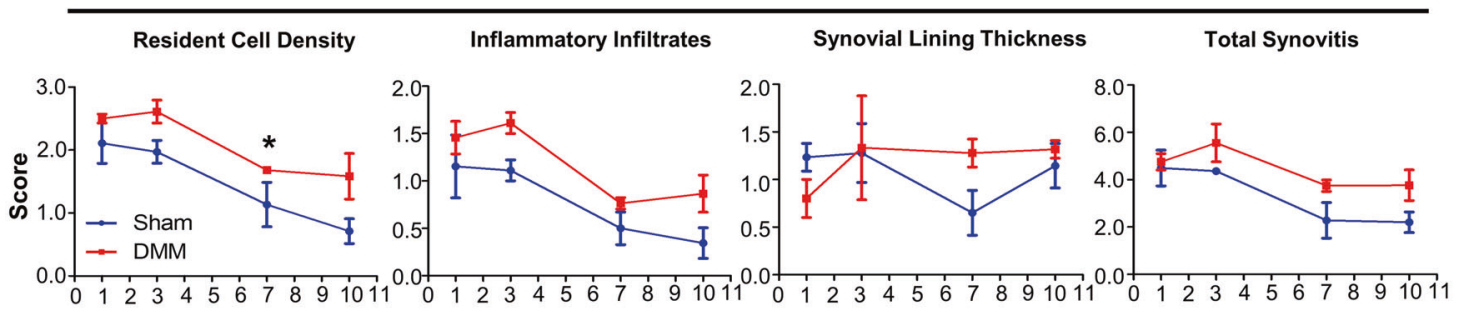

Posterior
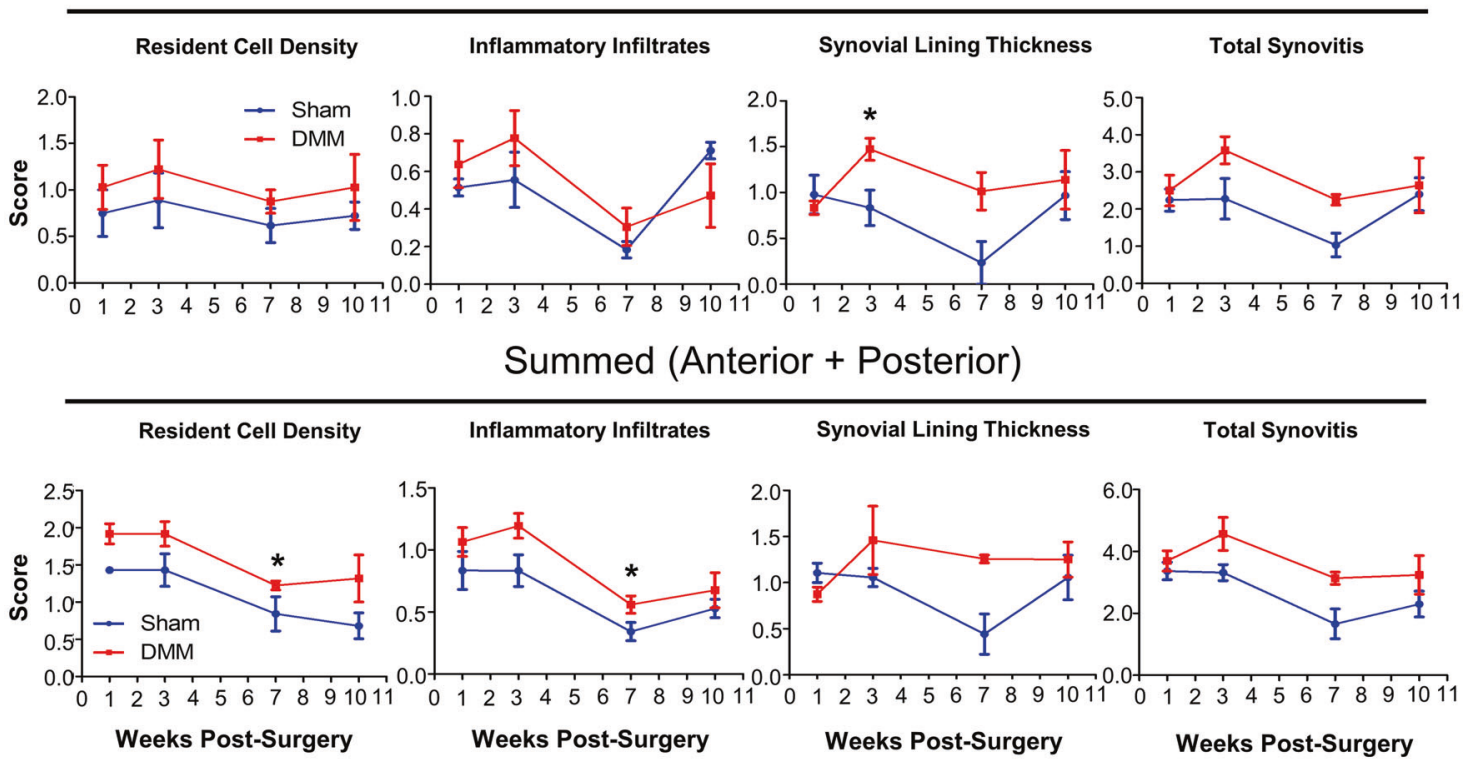

B

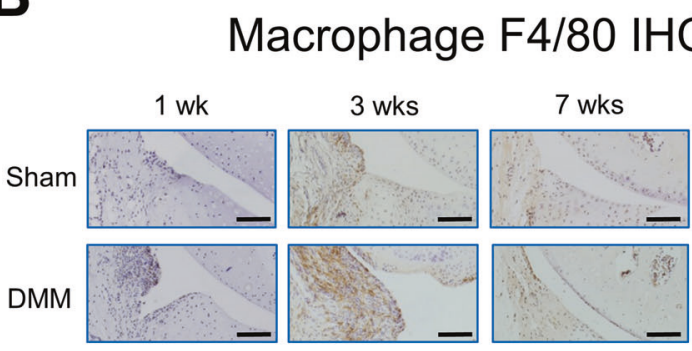

Fig. 2 Analysis of synovitis and macrophage presence in osteoarthritic mice. The synovium of $\mathrm{NF \kappa B}-\mathrm{RE}$-luc reporter mice were analyzed after DMM and sham surgery at 1,3,7, and 10 weeks. a Quantification of synovitis based on the following categories: resident cell density, presence of inflammatory infiltrates, and synovial lining thickness. Anterior and posterior synovitis were scored separately and summed for total score. Kruskal-Wallis statistical analysis followed by Dunn's test was conducted. b Images of immunohistochemical (IHC) analysis

inflammatory cells, and the thickness of the synovial lining. Our results showed that in the first 3 weeks following surgery in both the DMM and sham joints, resident cell density was higher and there were more inflammatory infiltrates than at subsequent weeks (Fig. 2a), an observation consistent with an early phase of inflammation that eventually resolves. Additionally, the DMM joint generally
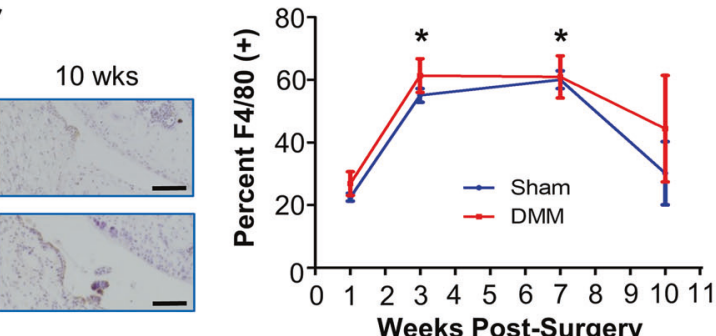

using the F4/80 antibody to detect resident and activated macrophages. Scale bar $=50 \mu \mathrm{m}$. c Quantification of macrophages detected from IHC images in (b). At least 3 mice were used for each time point. Values at 1 week post-DMM were used as a reference to be compared with values from the DMM samples at subsequent time points in statistical analysis. ANOVA analysis was conducted, followed by Bonferroni's post-hoc test. Asterisk $(*)$ denotes $p \leq 0.05$

had higher resident cell density, a greater amount of inflammatory infiltrates, and a thicker synovial lining compared to sham (Fig. 2a). Furthermore, the anterior region of the synovium had higher synovitis scores than the posterior region, which is likely related to the fact that the open knee surgery took place in the anterior area of the joint (Fig. 2a) [39]. 
Fig. 3 Histological assessment of joint structure damage in osteoarthritic mice. a Safranin $\mathrm{O} /$ Fast Green staining of mouse knee sections at $1,3,7$, and 10 weeks post-surgery, indicating structural changes and matrix loss. b Cartilage structure and matrix loss were quantified using the modified Mankin scoring system. Separate scores were obtained for the femur and the tibia, and these scores were combined as "total scores".

Values at 1 week post-DMM were used as a reference to be compared with values at subsequent time points in statistical analysis. At least 3 mice were used for each time point. Kruskal-Wallis statistical analysis was conducted,

followed by Dunn's test. Asterisk $(*)$ denotes $p \leq 0.05$. c Collagen II staining of mouse knees joints at 1, 3, 7, and 10 weeks post-surgery. DAPI staining served as a counter staining to indicate nuclei. Images of collagen II IHC and DAPI staining were overlaid. Scale bar $=400 \mu \mathrm{m}$

A
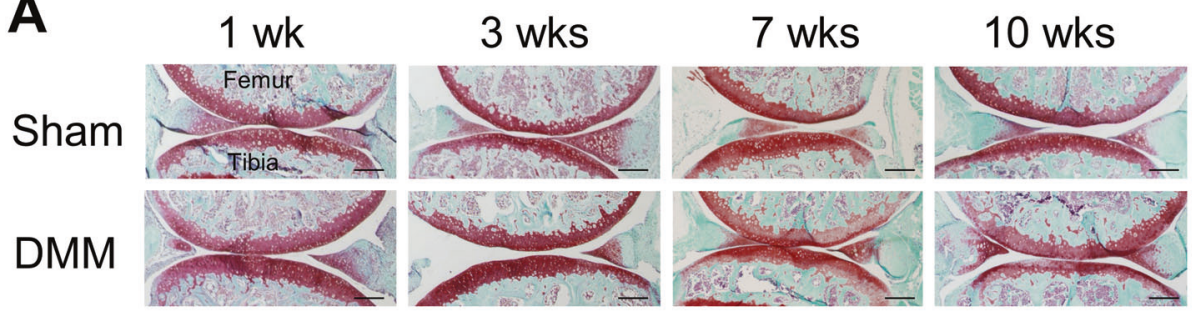

B Femur Tibia

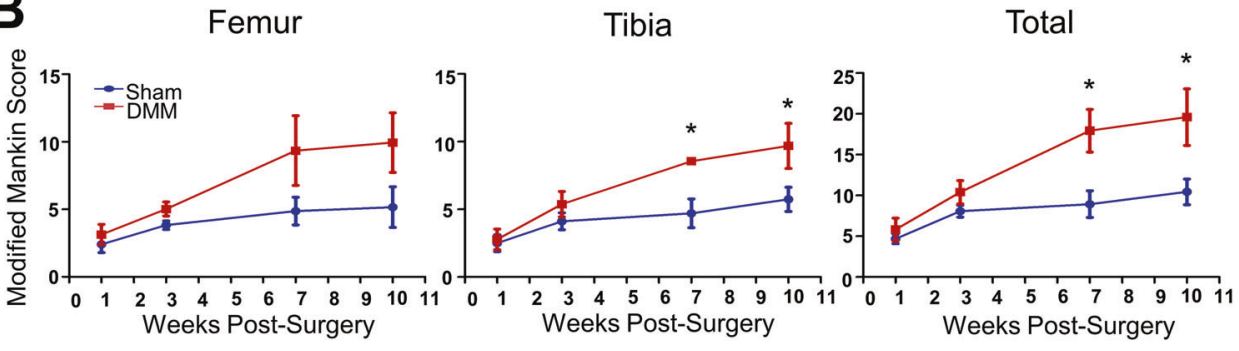

C

Sham

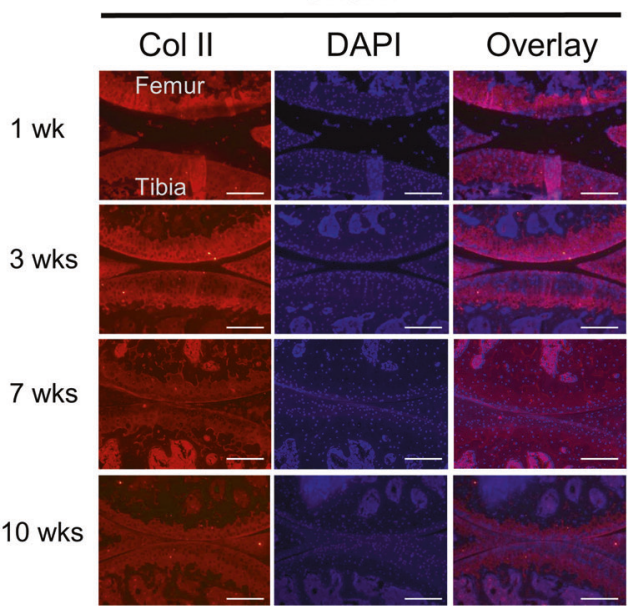

DMM

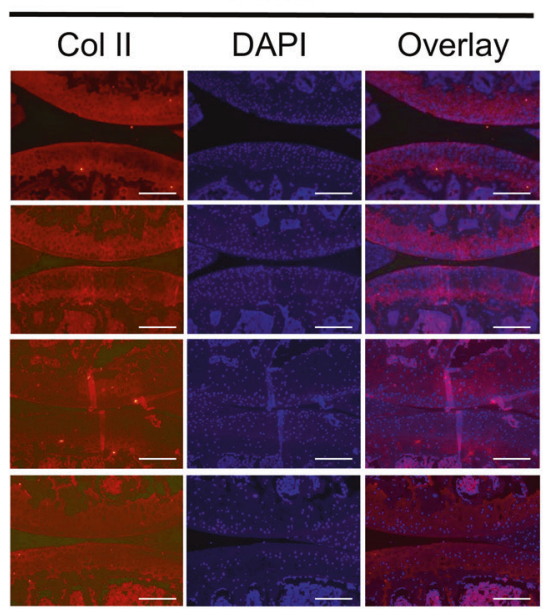

Since macrophages are known to contribute significantly to joint inflammation, we evaluated macrophage presence in the synovium after OA induction. We used an F4/80 antibody that broadly recognizes activated resident and infiltrating macrophages [63]. In both the DMM and the sham joints, there were significant increases in macrophage presence at 3 weeks and 7 weeks post-surgery, but no significant difference was noted for macrophage presence in the synovium between the sham and DMM joints (Fig. 2b, c). This result indicates that there is a delay in the surge of macrophage population compared to the NFKB activity and inflammatory infiltrates following surgery.

\section{Progressive GAG loss and articular structural damage in the DMM joint}

To investigate the cartilage structural loss following DMM surgery, we performed Safranin O staining to evaluate GAG content and assessed cartilage structural integrity by examining articular surface alterations such as fibrillation and clefts. Cartilage damage, reflected by the loss of surface integrity and Safranin O staining, was then quantified using the established modified Mankin system, where a higher score indicates increased damage [42]. As expected, a progressive increase in joint cartilage destruction was observed in the DMM knees, but not the sham knees (Fig. 3a, b). By 7 weeks and 10 weeks post-surgery, the DMM knees showed much higher scores in contrast to sham, indicating significant cartilage damage (Fig. 3a, b). In addition to GAGs, collagen II is a major matrix component in articular cartilage [64]. Thus, we evaluated collagen II protein levels via immunohistochemistry (IHC) (Fig. 3c, and Supplemental Fig. 1 for "no primary antibody" control). While no obvious differences in collagen II protein levels were found between the DMM and sham joints (Fig. 3c), further detailed quantification of collagen II protein level is 


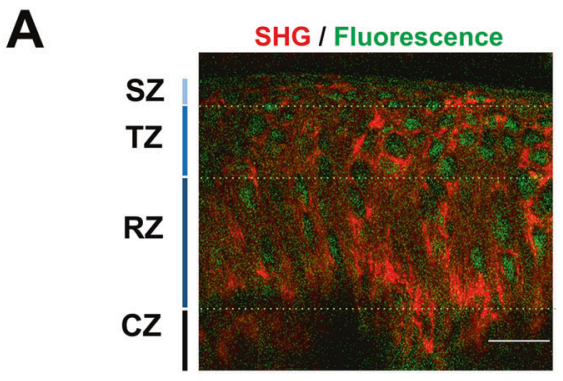

B

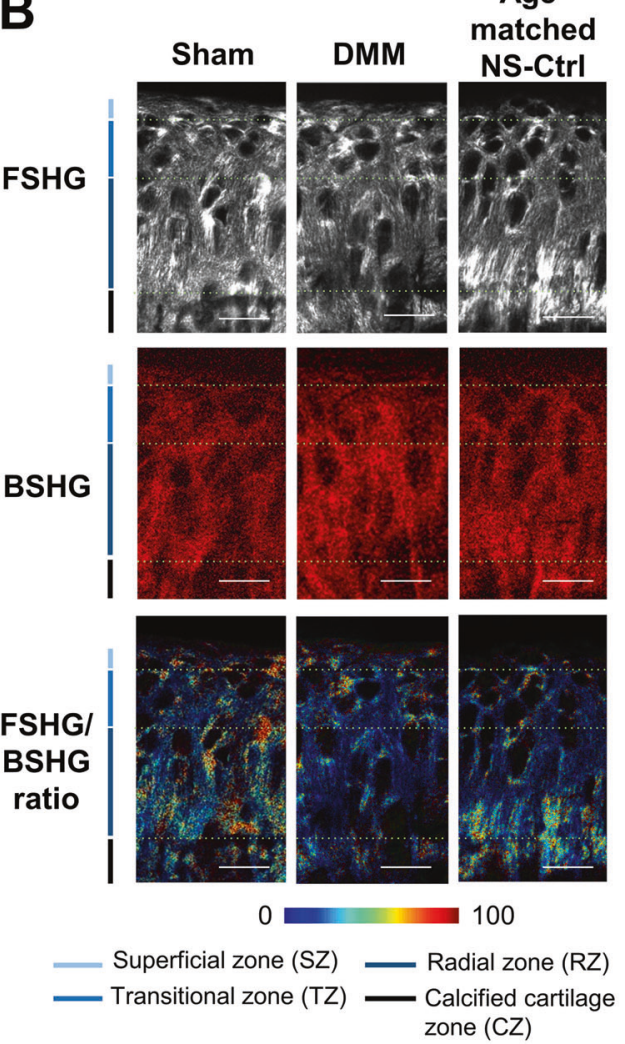

C
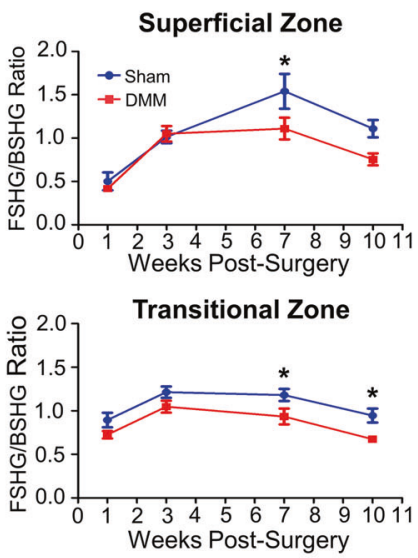

Radial Zone

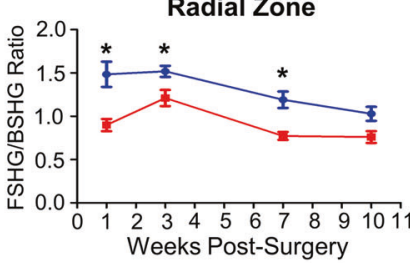

D
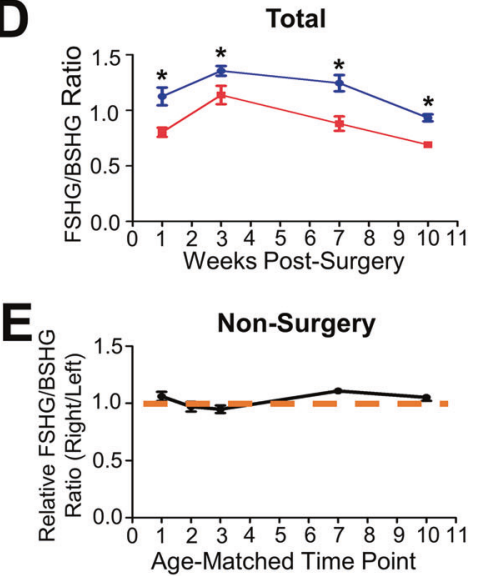

Fig. 4 Second Harmonic Generation (SHG) imaging assessment of collagen fiber thickness in osteoarthritic mice. Cartilage of the tibial plateau of NFkB-RE-luc reporter mice were analyzed after DMM and sham surgery at $1,3,7$, and 10 weeks. a Illustration of the delineation of various zones after SHG imaging. SZ: superficial zone, TZ: transitional zone, RZ: radial zone, CZ: calcified cartilage zone. b Representative images of the forward signal (FSHG), backward signal (BSHG), and forward/backward signal ratio (FSHG/BSHG) acquired for DMM, sham, and non-surgery control samples from 3 weeks post-

needed. It has been widely reported that in late OA, fibrosis takes place; the development of fibrosis is exemplified by the appearance of collagen I protein, which is not normally present in articular cartilage [65, 66]. Although collagen I protein was present in the bone and lateral meniscus in our IHC analysis, no collagen I was observed in the articular surface of the DMM joint up until 10 weeks post-surgery; however, collagen I protein was clearly present in the articular surface at 21 weeks post-surgery (Supplemental surgery. c Quantification of FSHG/BSHG signal ratios in the superficial, transitional, and radial zones of DMM and sham joints. At least 5 mice were used for each time point. d Quantification of average FSHG/BSHG signal for all zones in DMM and sham joints. e Quantification of average FSHG/BSHG signal for all zones in age-matched non-surgery controls. Data is presented as right knee signal relative to left knee signal. Comparisons were made between DMM and sham values using ANOVA statistical analysis followed by Bonferroni's post-hoc test. Asterisk $(*)$ denotes $p \leq 0.05$. Scale bars $=25 \mu \mathrm{m}$

Fig. 2). Thus, our data suggests that fibrosis is not present in early OA, but does take place in late OA, which is consistent with prior observations [65].

\section{Second Harmonic Generation imaging indicates reduced collagen fiber thickness in early $\mathrm{OA}$}

In addition to collagen content, collagen structure is known to change in late OA $[14,36,67]$. Second harmonic 
A

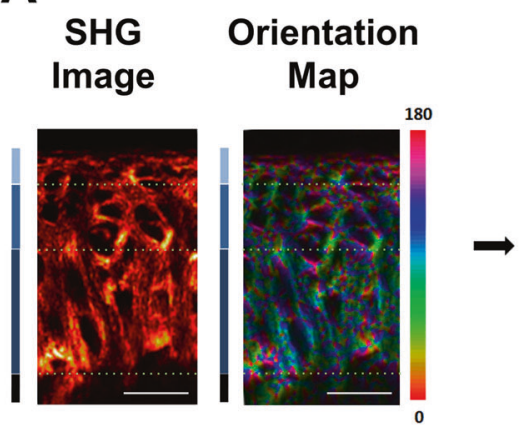

C

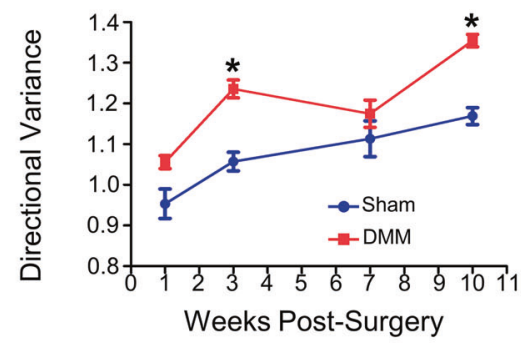

D

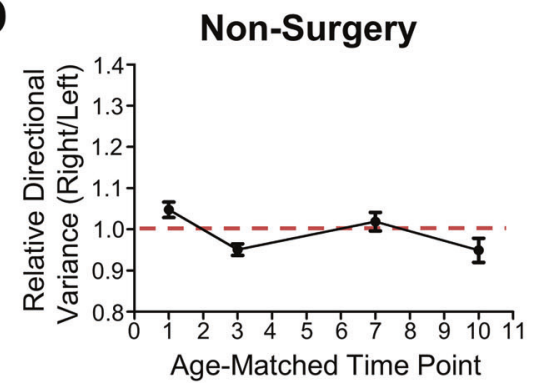

Directional Variance Map

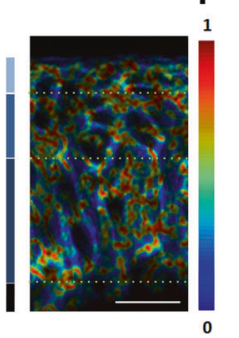

B
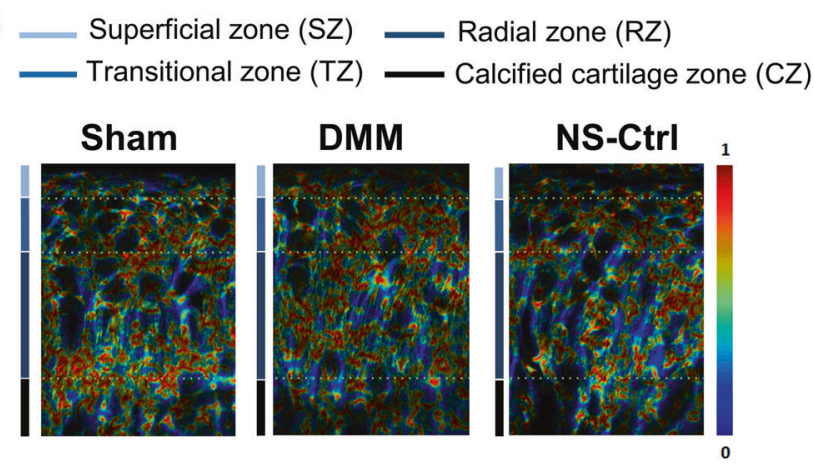
$(\mathrm{CZ})$
Transitional Zone

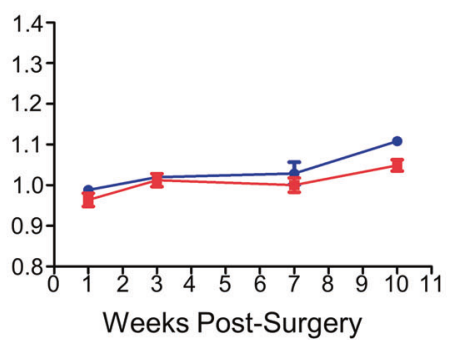

Non-Surgery

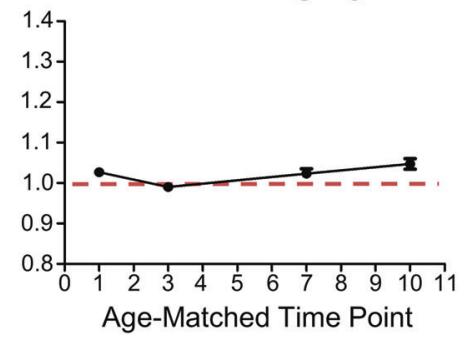

Radial Zone

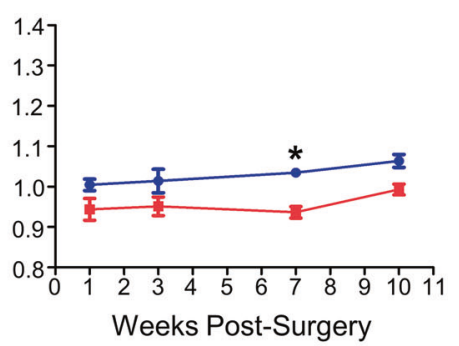

Non-Surgery

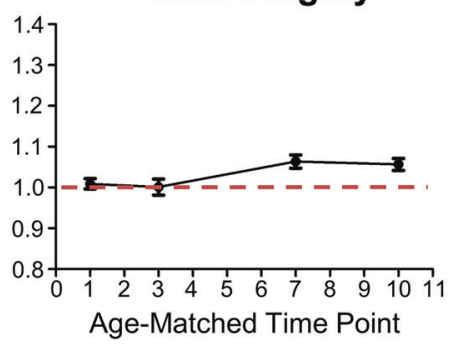

Fig. 5 Second Harmonic Generation (SHG) imaging assessment of collagen fiber orientation in osteoarthritic mice. Cartilage of the tibial plateau of NFKB-RE-luc reporter mice were analyzed after DMM and sham surgery at $1,3,7$, and 10 weeks. a Illustration of analysis on SHG image to calculate the directional variance of collagen fibers. Scale bar $=25 \mu \mathrm{m}$. b Representative images of the directional variance heat maps for sham, DMM, and age-matched non-surgery control. c
Quantification of collagen fiber directional variance in the superficial, transitional, and radial zones. At least 5 mice were used for each time point. d Quantification of directional variance of collagen fibers for age-matched non-surgery controls (right knee/ left knee) in the superficial, transitional, and radial zones. Comparisons were made between DMM and sham values using ANOVA statistical analysis followed by Bonferroni's post-hoc test. Asterisk (*) denotes $p \leq 0.05$ generation (SHG) imaging is widely accepted as an advanced approach to resolve fine structures of noncentrosymmetric structures such as fibrillar collagen [68, 69]. Thus, we performed SHG imaging to investigate changes in collagen fibers in OA progression. To visualize chondrocytes, we performed two-photon excited fluorescence microscopy imaging at the same time, in which endogenous fluorescence from the coenzyme FAD could be obtained, indicating cells within the matrix [48, 49, 53-56] (Fig. 4a). Since different zones of articular cartilage (superficial, transitional, and radial) consist of collagen fibers with different thicknesses [53-56], we analyzed fiber thickness in distinct zones. These zones were delineated based on their different alignment of collagen fibers and chondrocytes observed under the microscope (Fig. 4a). $H \& E$ staining confirmed the presence of all three zones in the DMM, indicating the mildness of OA in these early stages (Supplemental Fig. 3). Based on established works that assessed collagen fiber thickness using SHG imaging [9, 37, 68], we calculated the ratio of the SHG signal collected in forward (FSHG) and backward (BSHG) directions. Thicker collagen fibers result in significantly increased FSHG signals as compared to the BSHG signals [17]. Then, based on the forward SHG images and backward SHG images of articular cartilage, we generated FSHG/BSHG signal ratio maps (Fig. 4b).

Our results show that there were significant changes of the FSHG/BSHG ratio in the DMM and sham joint cartilage 
at multiple time points (Fig. 4c, d), while the FSHG/BSHG ratio in the cartilage of the non-surgery joint remained the same over time (Fig. 4e). This suggests that knee surgery altered collagen fiber thickness. Interestingly, there were no differences in the FSHG/BSHG ratio in the superficial and transitional zones prior to 7 weeks post-surgery, but the FSHG/BSHG ratios of the DMM knees in the radial zone were significantly different than the sham at 1,3 , and 7 weeks post-surgery (Fig. 4c). When all articular cartilage zones were considered, the FSHG/BSHG ratios of the DMM knees were lower than those of the sham knees at all time points (Fig. 4d), suggesting that the collagen fibers were thinner after DMM surgery. This trend started from the deep zone early in OA development, and extended through all the zones as OA progressed.

\section{Second Harmonic Generation imaging indicates increased variability of collagen fiber orientation in early $O A$}

We next evaluated collagen fiber orientation in the DMM and sham joints. First, the angle of the fibers in reference to the articular surface was determined using a previously established method [48, 57], with $0^{\circ}$ and $180^{\circ}$ being parallel to the surface, and $90^{\circ}$ being perpendicular to the surface (Fig. 5a). Consistent with published data [48, 70, 71], SHG showed clear differences in fiber orientation in the three zones of articular cartilage. In the superficial zone, the hues of collagen were mainly red or magenta, revealing that the majority of these fibers aligned parallel to the surface. In the radial zone, the fibers were mainly blue and green, showing that these fibers are typically perpendicular to the surface. In the transitional zone, the hues of the fibers were broadly distributed, indicating more randomly oriented fibers (Fig. 5a). To compare the state of collagen fiber orientation among different cartilage layers, we calculated the "directional variance" [48]. This parameter measures the variability of fiber orientation with respect to neighboring fibers, indicating the spread of the data (Fig. 5a). A higher directional variance value indicates a higher variance in collagen fiber orientation and more disorganized fibers, while a lower directional variance value indicates fibers that are more aligned with respect to each other [48]. This is reflected by the "directional variance heat map" (Fig. 5a). Representative images of "directional variance heat maps" for the sham, DMM, and non-surgery control samples are shown in Fig. $5 b$.

Both the superficial and radial zones showed notable differences at multiple time points in the directional variance of collagen fibers between the sham and DMM joints; however, no significant difference in directional variance was found in the transitional zone, or between the nonsurgery left and right knees (Fig. 5c,d). In the superficial zone, the DMM joint showed significantly higher directional variance of collagen fibers compared to that of the sham joint, and increased over time (Fig. 5c). Interestingly, we detected opposite changes in the radial zone, where the DMM joint showed decreased directional variance relative to the sham joint (Fig. 5c), suggesting that the collagen fibers in the deep zone became more organized in OA. Thus, changes in collagen fibers take place not only in the superficial layer but also in the deep layer of the articular cartilage during early OA development.

We further confirmed the authenticity of SHG imaging in reflecting collagen II content by using sections stained with an anti-collagen II antibody, since collagen II is the major collagen in articular cartilage [72, 73]. Confocal imaging of Col II immunofluorescence signals showed agreement of collagen II fiber signals with SHG imaging (Supplemental Fig. 4), supporting the notion that collagen II fibers are a major contributor to SHG signals. However, confocal imaging was not able to resolve fine details of collagen fiber alignment, unlike SHG imaging (Supplemental Figs. 5-6). This is probably related to the fact that confocal imaging relies on indirect signals from exogenous proteins (i.e., antibodies) binding to the collagen proteins; uneven binding of antibodies will result in distortion of the shape of the fibers as viewed by confocal imaging. In contrast, SHG imaging relies directly on signals from the endogenous collagen fibers, and truthfully reflects the collagen landscape.

\section{Discussion}

In this study, we used advanced imaging analyses to uncover novel pathological changes in the joint in early phases of experimental OA, including inflammation and zonal collagen structural alterations. Bioluminescence imaging analysis using NFkB-RE-luc reporter mice indicated an initial surge in inflammation that gradually resolved over time; but compared to the sham, OA mice showed a significant delay in resolution of inflammation. Although NFKB activity eventually returned to the basal level in our study, it is not clear whether its activity is altered when OA becomes even more severe. In human radiographic $\mathrm{OA}$, which likely represents stages later than those in our study, IHC analysis still detected a significantly higher level of synovial NFKB, indicating chronic inflammation [74]. Chronic inflammation is known to associate with pain in multiple tissues [75, 76]. One study used a pain OA model, monosodium iodoacetate (MIA) injection, that shows acute joint destruction. There, a surge of NFKB activity was observed immediately after MIA injection, accompanied by an immediate increase in pain [59]. We focused on early pathological changes prior to pain 
development during chronic OA development using the DMM model, in which pain only developed 12 weeks later [77]. But based on the demonstrated association of NFKB activity with pain, it will be interesting to correlate early $\mathrm{NF \kappa B}$ activity with the level of pain developed at later stages in chronic OA.

Our detailed synovitis analysis corroborated the NFkB imaging analysis. Regarding regions of the synovium that exhibit inflammatory changes, one prior study evaluated overall synovitis in DMM mice, and showed a higher level of synovitis in the anterior compartment [78]. Our data is consistent with this report; this finding is not entirely surprising, since the ligament severed in the DMM surgery is located at the anterior area of the joint [39]. However, there is a paucity of prior studies that separately analyzed various contributors of synovitis in different compartments of the joint during early OA progression. Our data showed an intriguing dynamic of synovitis in the DMM joint, which is mostly driven by cell density and inflammatory infiltrates, rather than the thickness of synovial lining. Despite that, synovial lining thickness was overall higher in the DMM joint. It is not clear how levels of these three contributors to synovitis will change in later stages in our system. But at least in humans, a study showed that the amounts of inflammatory infiltrates actually increased in late OA compared to early radiographic OA, suggesting inflammatory cells may participate in the manifestation of chronic pathogenesis [74]. Since macrophages have also been known to be present in OA, we examined the presence of activated macrophages by performing F4/80 staining. The level of activated macrophages was low at 1 week postsurgery, but significantly increased afterward. Interestingly, the trend of activated macrophage presence did not follow the same trend as synovitis. On the other hand, since the F4/ 80 antibody that we used can detect pro-inflammatory macrophages (M1), pro-resolution macrophages (M2), and synovial resident macrophages, the dynamic presence of each type of macrophages is still unclear [79-82]. Thus, further studies are needed to determine the specific contributions of different subpopulations of inflammatory cells in early OA. Nevertheless, since inflammation is known to induce cartilage-degrading enzymes such a MMPs, it is possible that this prolonged period of inflammation sustains catabolic activities to a point of irreversibility, constituting a critical window for OA progression.

Profiles of cartilage structural changes are also dynamic. In the early stages of OA, we found structural damage, such as fibrillation and clefts, was relatively trivial, which is consistent with other studies [40, 83]. It has been generally regarded that collagen experiences fewer changes in OA than GAGs, based on the ${ }^{14} \mathrm{C}$ labeling analysis of human cartilage that indicated a higher turnover rate of GAGs than collagen [14, 84]. By using SHG imaging, however, we discovered that collagen organization did in fact change rapidly upon OA initiation, beginning from the earliest time point post DMM surgery and in different layers of the articular cartilage, reflecting the dynamics of ECM during OA pathogenesis.

In terms of collagen fiber thickness, at 1 week postsurgery, collagen fibers in the radial zone were already thinner, while collagen fibers in the superficial and transitional zones did not become thinner until 7 weeks postsurgery, suggesting that the change in fiber thickness in cartilage matrix begins in the deep layer rather than the superficial layer during OA progression. While no reports on collagen fiber thickness have been carried out at very early OA time points, as in our work, previous studies examined later stages of OA. In these studies, both an increase and decrease in collagen fiber thickness was found $[7,8,37,85-87]$. The discrepancy could be due to the balance of damaged collagen II fibers naturally found in articular cartilage and the occurrence of collagen I fibers, which are thicker than collagen II fibers, in later stage OA [88]. In the time frame of our study (1 to 10 weeks), however, no collagen I expression was detected (Supplemental Fig. 2). Thus, we do not believe that fibrosis interfered with our interpretation of the SHG signals. Collagen X is another collagen type that has also been found to be elevated in OA articular cartilage $[89,90]$. However, since collagen $\mathrm{X}$ is not a fibrillar collagen, it does not interfere with SHG signal interpretation [72, 73]. Additionally, we did not observe an increase in collagen $\mathrm{X}$ in the time frame of our study, which is consistent with the study indicating collagen $\mathrm{X}$ upregulation only takes place in later stages of OA (Supplemental Fig. 7) [90].

While changes in collagen fiber thickness may first take place in the deep zone, changes in collagen orientation seem to first take place in the superficial zone, showing an upward trajectory in directional variance following DMM surgery. This study is consistent with prior studies on human cartilage using polarized light microscopy and on sheep cartilage using MRI [14, 91], and supports the hypothesis that the mouse DMM model recapitulates collagen structural changes in human OA, even though these prior studies were focused on later time points of OA pathogenesis. A recent report using AFM-based nanoindentation demonstrated a significant reduction of mouse cartilage modulus in the superficial zone starting from 1 week post-DMM surgery [92]. However, the underlying cause for this reduction is not known. Our study provides a mechanistic explanation for the change in biomechanical properties of cartilage matrix in OA, which may be caused by a combination of altered directional variance and thickness in collagen fibers. Contrary to the superficial zone, however, the deep zone showed more organized collagen fibers in OA (Fig. 6). Whether this change results 


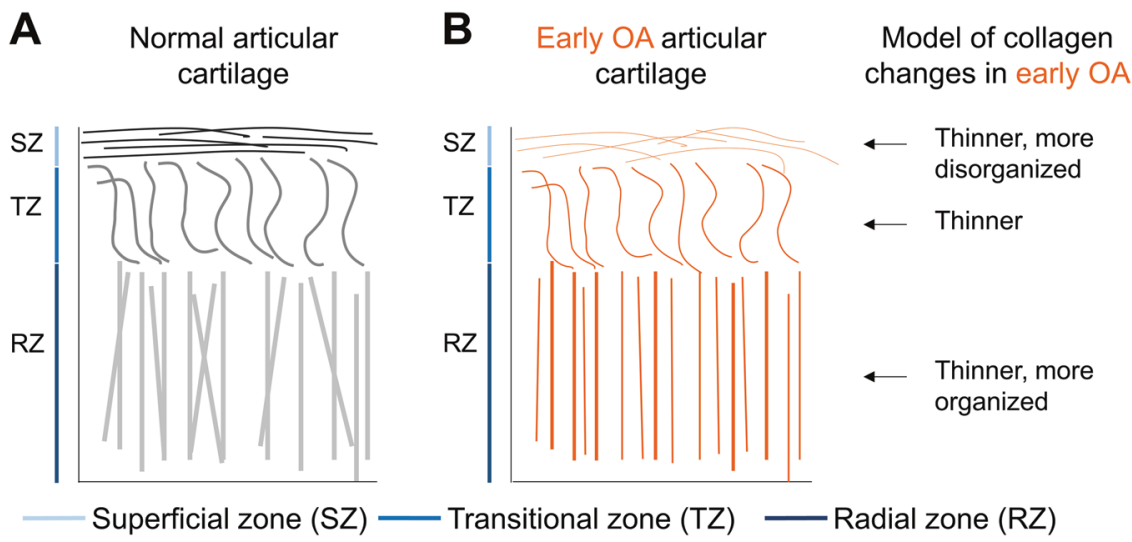

Fig. 6 Model of collage fiber changes in articular cartilage in early OA development. a Normal articular cartilage consists of three zones: the superficial zone, where collagen fibers lie parallel to the articular surface; the transitional zone, where collagen fibers are sinusoidal; and

in alteration of biomechanical properties of the deep zone remains to be discovered. The notion of inhomogeneity of articular cartilage has previously been raised concerning normal cartilage [93]. Here, we showed that inhomogeneity is also present in collagen fibers under the pathological condition of OA, and may even exhibit opposite trends in different layers.

The dynamic profiles of joint inflammation and collagen structure changes in our OA studies suggest that cartilage structural changes may be independent of inflammatory changes at the onset of the disease. Specifically, joint inflammation and synovitis were equally pronounced in the DMM and sham joints at the first week post-OA induction surgery; at the same time points, collagen fibers were already thinner and more disorientated. On the other hand, this does not exclude the possibility that the prolonged inflammatory phase that follows sustains the collagen fiber changes over the course of early OA development, due to the gradual induction of MMP activities. In fact, in humans, synovitis strongly correlates and predicts human radiographic OA [94-96]. Interestingly, we found an association between the trend of collagen fiber thickness changes in the deep zone with that of synovitis in both the DMM and Sham knees; however, DMM surgery leads to an overall shift toward higher synovitis and thinner collagen fibers (Supplemental Fig. 8A). It is also interesting to note that the trend of collagen directional variance in the superficial zone follows that of the Mankin score in OA progression, indicating a coordinated change between different components of cartilage matrix (Supplemental Fig. 8B). As the biological rationale behind such observations is still elusive, it is too early to conclude whether such correlation is causative or not.

In summary, by using the bioluminescence imaging and SHG imaging approaches, we were able to take a first look the radial zone, where collagen fibers lie perpendicular to the articular surface. b Model of collagen fiber landscape alterations in early OA: collagen fiber thinning and changes in directional variance across all zones of articular cartilage

at inflammatory activities and detailed collagen structural changes in $\mathrm{OA}$ at much earlier time points than previously reported and obtained significant and intriguing results. While SHG imaging has been used to visualize collagen in cartilage [34-38, 97], our study uniquely quantified the properties of collagen fiber thickness and directional organization. The sensitive nature of these imaging approaches will not only be powerful for evaluating early pathology in other subtypes of OA or in human OA specimens, but it will also enable us to assess changes in the joint more effectively to evaluate interventions in developing $\mathrm{OA}$ treatment options.

Acknowledgements We thank the Tufts Small Animal Imaging Facility for their support with in vivo imaging. We are also thankful to Dr. David Rowe (University of Connecticut) and his lab for sharing their cryosectioning technique with cryofilm. Finally, we are thankful to all members of the Zeng laboratory for their helpful discussions.

\section{Compliance with ethical standards}

Conflict of interest TEM serves as a consultant for Sanofi Aventis, Flexion Therapeutics, and Seikogaku. TEM also serves on the advisory and safety monitoring board of Samumed, Pfizer, Astellas, and Regeneron. Additionally, TEM lectures at Orthogen. The remaining authors declare that they have no conflict of interest.

\section{References}

1. Bitton R. The economic burden of osteoarthritis. Am J Manag Care. 2009 Sep;15(8 Suppl):S230-235.

2. Le TK, Montejano LB, Cao Z, et al. Health care costs in US patients with and without a diagnosis of osteoarthritis. J Pain Res. 2012;5:23-30.

3. Le TK, Montejano LB, Cao Z, et al. Healthcare costs associated with osteoarthritis in US patients. Pain Pract Off $\mathrm{J}$ World Inst Pain. 2012;12(8):633-40. 
4. Poole AR. Osteoarthritis as a whole joint disease. HSS J. 2012 Feb;8(1):4-6.

5. Loeser RF, Goldring SR, Scanzello CR, et al. Osteoarthritis: a disease of the joint as an organ. Arthritis Rheum. 2012;64:1697-707.

6. Novakofski KD, Pownder SL, Koff MF, et al. High-resolution methods for diagnosing cartilage damage in vivo. Cartilage. 2016;7(1):39-51.

7. Desrochers J, Amrein MW, Matyas JR. Viscoelasticity of the articular cartilage surface in early osteoarthritis. Osteoarthritis Cartilage. 2012;20:413-21.

8. Thambyah A, Zhao J-Y, Bevill SL, et al. Macro-, micro-, and ultrastructural investigation of how degeneration influences the response of cartilage to loading. J Mech Behav Biomed Mater. 2012;5(1):206-15.

9. Chu CR, Williams AA, Coyle $\mathrm{CH}$, et al. Early diagnosis to enable early treatment of pre-osteoarthritis. Arthritis Res Ther. 2012;14 (3):212.

10. Lim NH, Vincent TL, Nissim A. In vivo optical imaging of early osteoarthritis using an antibody specific to damaged arthritic cartilage. Arthritis Res Ther. 2015;17:376.

11. Casula V, Nissi MJ, Podlipská J, et al. Elevated adiabatic T1 $\rho$ and T2 $\rho$ in articular cartilage are associated with cartilage and bone lesions in early osteoarthritis: A preliminary study. J Magn Reson Imaging JMRI. 2017;46(3):678-89.

12. Wirth W, Maschek S, Eckstein F. Sex- and age-dependence of region- and layer-specific knee cartilage composition (spin-spinrelaxation time) in healthy reference subjects. Ann Anat Anat Anz Off Organ Anat Ges. 2017;210:1-8.

13. Nia HT, Gauci SJ, Azadi M, et al. High-bandwidth AFM-based rheology is a sensitive indicator of early cartilage aggrecan degradation relevant to mouse models of osteoarthritis. J Biomech. 2015;48(1):162-5.

14. Saarakkala S, Julkunen P, Kiviranta $P$, et al. Depth-wise progression of osteoarthritis in human articular cartilage: investigation of composition, structure and biomechanics. Osteoarthr Cartil OARS Osteoarthr Res Soc. 2010;18(1):73-81.

15. Rieppo J, Hyttinen MM, Halmesmaki E, et al. Changes in spatial collagen content and collagen network architecture in porcine articular cartilage during growth and maturation. Osteoarthr Cartil OARS Osteoarthr Res Soc. 2009;17(4):448-55.

16. Hyttinen MM, Arokoski JP, Parkkinen JJ, et al. Age matters: collagen birefringence of superficial articular cartilage is increased in young guinea-pigs but decreased in older animals after identical physiological type of joint loading. Osteoarthritis Cartilage. 2001;9:694-701.

17. Chu S-W, Tai S-P, Chan M-C, et al. Thickness dependence of optical second harmonic generation in collagen fibrils. Opt Express. 2007;15(19):12005-10.

18. Zhang Q, Li H, Zhang Z, et al. F. Serum metabolites as potential biomarkers for diagnosis of knee osteoarthritis. Dis Markers. 2015;2015:684794

19. Yarmola EG, Shah Y, Arnold DP, et al. Magnetic capture of a molecular biomarker from synovial fluid in a rat model of knee osteoarthritis. Ann Biomed Eng. 2016;44(4):1159-69.

20. Frisbie DD, Al-Sobayil F, Billinghurst RC, et al. Changes in synovial fluid and serum biomarkers with exercise and early osteoarthritis in horses. Osteoarthritis Cartilage. 2008;16:1196-204.

21. Friel NA, Chu CR. The role of ACL injury in the development of posttraumatic knee osteoarthritis. Clin Sports Med. 2013 Jan;32 (1): $1-12$.

22. Rosenberg GA. Extracellular matrix inflammation in vascular cognitive impairment and dementia. Clin Sci Lond Engl. 2017;131:425-37.
23. Suthahar N, Meijers WC, Silljé HHW, et al. From Inflammation to fibrosis-molecular and cellular mechanisms of myocardial tissue remodelling and perspectives on differential treatment opportunities. Curr Heart Fail Rep. 2017;14:235-50.

24. Wight TN, Frevert CW, Debley JS, et al. Interplay of extracellular matrix and leukocytes in lung inflammation. Cell Immunol. 2017;312:1-14.

25. Goldring MB, Otero M, Tsuchimochi K, et al. Defining the roles of inflammatory and anabolic cytokines in cartilage metabolism. Ann Rheum Dis. 2008;67(Suppl 3):75-82.

26. Wojdasiewicz P, Poniatowski $Ł$, Szukiewicz D. The role of inflammatory and anti-inflammatory cytokines in the pathogenesis of osteoarthritis. Mediators Inflamm. https://www.hindawi.com/ journals/mi/2014/561459/abs/.

27. Glasson SS, Askew R, Sheppard B, Carito B, Blanchet T, Ma HL, et al. Deletion of active ADAMTS5 prevents cartilage degradation in a murine model of osteoarthritis. Nature. 2005;434 (7033):644-8.

28. Goldring MB, Otero M, Plumb DA, et al. Roles of inflammatory and anabolic cytokines in cartilage metabolism: signals and multiple effectors converge upon MMP-13 regulation in osteoarthritis. Eur Cell Mater. 2011;21:202-20.

29. Lieberthal J, Sambamurthy N, Scanzello CR. Inflammation in joint injury and post-traumatic osteoarthritis. Osteoarthritis Cartilage. 2015;23:1825-34.

30. Irie K, Uchiyama E, Iwaso H. Intraarticular inflammatory cytokines in acute anterior cruciate ligament injured knee. The Knee. 2003;10:93-6.

31. van Meegeren MER, Roosendaal G, Jansen NWD, et al. IL-4 alone and in combination with IL-10 protects against blood-induced cartilage damage. Osteoarthritis Cartilage. 2012;20:764-72.

32. Helmark IC, Mikkelsen UR, Børglum J, et al. Exercise increases interleukin-10 levels both intraarticularly and peri-synovially in patients with knee osteoarthritis: a randomized controlled trial. Arthritis Res Ther. 2010;12:126.

33. Chen LX, Lin L, Wang HJ, et al. Suppression of early experimental osteoarthritis by in vivo delivery of the adenoviral vectormediated NF-kappaBp65-specific siRNA. Osteoarthr Cartil OARS Osteoarthr Res Soc. 2008;16(2):174-84.

34. Campagnola P. Second harmonic generation imaging microscopy: applications to diseases diagnostics. Anal Chem. 2011;83 (9):3224-31.

35. Matcher SJ. What can biophotonics tell us about the 3D microstructure of articular cartilage? Quant Imaging Med Surg. 2015;5:143-58.

36. Mansfield JC, Winlove CP, Moger J, et al. Collagen fiber arrangement in normal and diseased cartilage studied by polarization sensitive nonlinear microscopy. J Biomed Opt. 2008; $13: 44020$.

37. Houle M-A, Couture C-A, Bancelin S, et al. Analysis of forward and backward Second Harmonic Generation images to probe the nanoscale structure of collagen within bone and cartilage. J Biophotonics. 2015;8:993-1001.

38. Chen X, Nadiarynkh O, Plotnikov S, et al. Second harmonic generation microscopy for quantitative analysis of collagen fibrillar structure. Nat Protoc. 2012;7:654-69.

39. Glasson SS, Blanchet TJ, Morris EA. The surgical destabilization of the medial meniscus (DMM) model of osteoarthritis in the 129/ SvEv mouse. Osteoarthr Cartil OARS Osteoarthr Res Soc. 2007 Sep;15(9):1061-9.

40. Leahy AA, Esfahani SA, Foote AT, et al. Analysis of the trajectory of osteoarthritis development in a mouse model by serial near-infrared fluorescence imaging of matrix metalloproteinase activities. Arthritis Rheumatol Hoboken NJ. 2015;67(2):442-53. 
41. Gibson AL, Hui Mingalone CK, Foote AT, et al. Wnt7a inhibits IL-1 $\beta$ induced catabolic gene expression and prevents articular cartilage damage in experimental osteoarthritis. Sci Rep. 2017;7:41823.

42. McNulty MA, Loeser RF, Davey C, et al. A comprehensive histological assessment of osteoarthritis lesions in mice. Cartilage. 2011;2(4):354-63.

43. Mankin HJ, Johnson ME, Lippiello L. Biochemical and metabolic abnormalities in articular cartilage from osteoarthritic human hips. III. Distribution and metabolism of amino sugar-containing macromolecules. J Bone Joint Surg Am. 1981;63(1):131-9.

44. Furman BD, Strand J, Hembree WC, et al. Joint degeneration following closed intraarticular fracture in the mouse knee: A model of posttraumatic arthritis. J Orthop Res. 2007;25:578-92.

45. Krenn V, Morawietz L, Häupl T, et al. Grading of chronic synovitis--a histopathological grading system for molecular and diagnostic pathology. Pathol Res Pract. 2002;198:317-25.

46. Uchimura T, Foote AT, Smith EL, et al. Insulin-like growth factor ii (igf-ii) inhibits il-1 $\beta$-induced cartilage matrix loss and promotes cartilage integrity in experimental osteoarthritis. J Cell Biochem. 2015;116(12):2858-69.

47. Kurth TB, De Bari C. Immunostaining of skeletal tissues. Methods Mol Biol Clifton NJ. 2012;816:321-34.

48. Liu Z, Pouli D, Sood D, et al. Automated quantification of threedimensional organization of fiber-like structures in biological tissues. Biomaterials. 2017;116:34-47.

49. Abbott RD, Borowsky FE, Quinn KP, et al. Non-invasive assessments of adipose tissue metabolism in vitro. Ann Biomed Eng. 2016;44:725-32.

50. Bayan C, Levitt JM, Miller E, et al. Fully automated, quantitative, noninvasive assessment of collagen fiber content and organization in thick collagen gels. J Appl Phys. 2009;105:102042.

51. Quinn KP, Sullivan KE, Liu Z, et al. Optical metrics of the extracellular matrix predict compositional and mechanical changes after myocardial infarction. Sci Rep 2016. http://www.ncbi. nlm.nih.gov/pmc/articles/PMC5098140/

52. Yildirim M, Quinn KP, Kobler JB, et al. Quantitative differentiation of normal and scarred tissues using second-harmonic generation microscopy. Scanning. 2016;38:684-93.

53. Benninghoff A. Form und bau der gelenkknorpel in ihren beziehungen zur funcion. II. $\mathrm{Z}$ Zellforsch Mikrosk Anaomie. $1925 ; 2: 783-862$.

54. Korhonen RK, Herzog W. Depth-dependent analysis of the role of collagen fibrils, fixed charges and fluid in the pericellular matrix of articular cartilage on chondrocyte mechanics. J Biomech. 2008;41:480-5.

55. Choi JB, Youn I, Cao L, et al. Zonal changes in the threedimensional morphology of the chondron under compression: The relationship among cellular, pericellular, and extracellular deformation in articular cartilage. J Biomech. 2007;40:2596-603.

56. Greco F, Specchia N, Falciglia F, et al. Ultrastructural analysis of the adaptation of articular cartilage to mechanical stimulation. Ital J Orthop Traumatol. 1992;18:311-21.

57. Quinn KP, Georgakoudi I. Rapid quantification of pixel-wise fiber orientation data in micrographs. J Biomed Opt. 2013;18:46003.

58. Quinn KP, Golberg A, Broelsch GF, et al. An automated image processing method to quantify collagen fibre organization within cutaneous scar tissue. Exp Dermatol. 2015;24:78-80.

59. Bowles RD, Mata BA, Bell RD, et al. In vivo luminescence imaging of NF- $\mathrm{KB}$ activity and serum cytokine levels predict pain sensitivities in a rodent model of osteoarthritis. Arthritis Rheumatol Hoboken NJ. 2014;66(3):637-46.

60. Spengler ML, Kuropatwinski KK, Comas M, et al. Core circadian protein CLOCK is a positive regulator of NF- $\mathrm{KB}-$ mediated transcription. Proc Natl Acad Sci U S A. 2012;109:2457-65.
61. Badr C, Niers JM, Tjon-Kon-Fat L-A, et al. Real-time monitoring of NF-kappaB activity in cultured cells and in animal models. Mol Imaging. 2009;8:278-90.

62. Krenn V, Morawietz L, Burmester G-R, et al. Synovitis score: discrimination between chronic low-grade and high-grade synovitis. Histopathology. 2006;49:358-64.

63. Gordon S, Plüddemann A, Martinez Estrada F. Macrophage heterogeneity in tissues: phenotypic diversity and functions. Immunol Rev. 2014;262:36-55.

64. Eyre D. Collagen of articular cartilage. Arthritis Res. 2002;4 (1):30-5.

65. Tsai M-R, Chen C-H, Sun C-K. Third and second harmonic generation imaging of human articular cartilage. In 2009. https:// doi.org/10.1117/12.809968. p. 71831V-71831V-10.

66. Miosge N, Hartmann M, Maelicke C, et al. Expression of collagen type I and type II in consecutive stages of human osteoarthritis. Histochem Cell Biol. 2004;122:229-36.

67. Felka T, Rothdiener M, Bast S, et al. Loss of spatial organization and destruction of the pericellular matrix in early osteoarthritis in vivo and in a novel in vitro methodology. Osteoarthritis Cartilage. $2016 \mathrm{Jul} ; 24: 1200-9$.

68. Liu Z, Quinn KP, Speroni L, et al. Rapid three-dimensional quantification of voxel-wise collagen fiber orientation. Biomed Opt Express. 2015;6:2294-310.

69. Williams RM, Zipfel WR, Webb WW. Interpreting secondharmonic generation images of collagen I fibrils. Biophys J. 2005 Feb;88(2):1377-86.

70. Hughes LC, Archer CW, ap Gwynn I. The ultrastructure of mouse articular cartilage: collagen orientation and implications for tissue functionality. A polarised light and scanning electron microscope study and review. Eur Cell Mater. 2005;9:68-84.

71. Nieminen MT, Rieppo J, Töyräs J, et al. T2 relaxation reveals spatial collagen architecture in articular cartilage: a comparative quantitative MRI and polarized light microscopic study. Magn Reson Med. 2001;46:487-93.

72. Mouw JK, Ou G, Weaver VM. Extracellular matrix assembly: a multiscale deconstruction. Nat Rev Mol Cell Biol. 2014;15:771-85.

73. Sutmuller M, Bruijn JA, de Heer E. Collagen types VIII and X, two non-fibrillar, short-chain collagens. Structure homologies, functions and involvement in pathology. Histol Histopathol. 1997; 12:557-66.

74. Ostojic M, Soljic V, Vukojevic K, et al. Immunohistochemical characterization of early and advanced knee osteoarthritis by NF$\kappa B$ and iNOS expression. J Orthop Res. 2017;35:1990-7.

75. Niederberger E, Geisslinger G. The IKK-NF-kappaB pathway: A source for novel molecular drug targets in pain therapy? FASEB J. 2008;22:3432-42.

76. Shih R-H, Wang C-Y, Yang C-M. NF-kappaB signaling pathways in neurological inflammation: A mini review. Front Mol Neurosci. 2015;8:77.

77. Inglis JJ, McNamee KE, Chia S-L, et al. Regulation of pain sensitivity in experimental osteoarthritis by the endogenous peripheral opioid system. Arthritis Rheum. 2008;58:3110-9.

78. Jackson MT, Moradi B, Zaki S, et al. Depletion of proteaseactivated receptor 2 but not protease-activated receptor 1 may confer protection against osteoarthritis in mice through extracartilaginous mechanisms. Arthritis Rheumatol. 2014;66 (12):3337-48.

79. Fitzgibbons TP, Czech MP. Emerging evidence for beneficial macrophage functions in atherosclerosis and obesity-induced insulin resistance. J Mol Med Berl Ger. 2016;94:267-75.

80. Hall KL, Volk-Draper LD, Flister MJ, et al. New model of macrophage acquisition of the lymphatic endothelial phenotype. PloS ONE. 2012;7:31794. 
81. Misharin AV, Morales-Nebreda L, Mutlu GM, et al. Flow cytometric analysis of macrophages and dendritic cell subsets in the mouse lung. Am J Respir Cell Mol Biol. 2013;49:503-10.

82. Utomo L, Bastiaansen-Jenniskens YM. Verhaar J a. N, et al. Cartilage inflammation and degeneration is enhanced by proinflammatory (M1) macrophages in vitro, but not inhibited directly by anti-inflammatory (M2) macrophages. Osteoarthritis Cartilage. 2016;24(12):2162-70.

83. Loeser RF, Olex AL, McNulty MA, et al. Disease progression and phasic changes in gene expression in a mouse model of osteoarthritis. PloS ONE. 2013;8(1):54633.

84. Heinemeier KM, Schjerling P, Heinemeier J, et al. Radiocarbon dating reveals minimal collagen turnover in both healthy and osteoarthritic human cartilage. Sci Transl Med. 2016;8:346ra90.

85. Stolz M, Gottardi R, Raiteri R, et al. Early detection of aging cartilage and osteoarthritis in mice and patient samples using atomic force microscopy. Nat Nanotechnol. 2009;4:186-92.

86. Xu L, Flahiff CM, Waldman BA, et al. Osteoarthritis-like changes and decreased mechanical function of articular cartilage in the joints of mice with the chondrodysplasia gene (cho). Arthritis Rheum. 2003;48:2509-18.

87. Kumar R, Grønhaug KM, Romijn EI, et al. Polarization second harmonic generation microscopy provides quantitative enhanced molecular specificity for tissue diagnostics. J Biophotonics. 2015;8:730-9.

88. Lodish H, Berk A, Zipursky SL, et al. Collagen: The Fibrous Proteins of the Matrix 2000. http://www.ncbi.nlm.nih.gov/books/ NBK21582/

89. Kronenberg HM. Developmental regulation of the growth plate. Nature. 2003;423:332-6.

90. Oliveria SA, Felson DT, Reed JI, Cirillo PA, Walker AM. Incidence of symptomatic hand, hip, and knee osteoarthritis among patients in a health maintenance organization. Arthritis Rheum. 1995 Aug;38(8):1134-41.

91. Koff MF, Shah P, Pownder S, et al. Correlation of meniscal T2* with multiphoton microscopy, and change of articular cartilage T2 in an ovine model of meniscal repair. Osteoarthritis Cartilage. 2013;21:1083-91.

92. Doyran B, Tong W, Li Q, et al. Nanoindentation modulus of murine cartilage: a sensitive indicator of the initiation and progression of post-traumatic osteoarthritis. Osteoarthritis Cartilage. 2017;25:108-17.

93. McLeod MA, Wilusz RE, Guilak F. Depth-dependent anisotropy of the micromechanical properties of the extracellular and pericellular matrices of articular cartilage evaluated via atomic force microscopy. J Biomech. 2013;46:586-92.

94. Felson DT, Niu J, Neogi T, et al. Synovitis and the risk of knee osteoarthritis: the MOST Study. Osteoarthritis Cartilage. 2016;24:458-64.

95. Guermazi A, Hayashi D, Roemer FW, et al. Synovitis in knee osteoarthritis assessed by contrast-enhanced magnetic resonance imaging (MRI) is associated with radiographic tibiofemoral osteoarthritis and MRI-detected widespread cartilage damage: the MOST study. J Rheumatol. 2014;41:501-8.

96. Ayral X, Pickering EH, Woodworth TG, et al. Synovitis: a potential predictive factor of structural progression of medial tibiofemoral knee osteoarthritis -- results of a 1 year longitudinal arthroscopic study in 422 patients. Osteoarthritis Cartilage. 2005;13:361-7.

97. Kiyomatsu H, Oshima Y, Saitou T, et al. Quantitative SHG imaging in osteoarthritis model mice, implying a diagnostic application. Biomed Opt Express. 2015;6:405-20. 